\title{
Tableaux and plane partitions of truncated shapes (extended abstract)
}

\author{
Greta Panova ${ }^{1}$ \\ ${ }^{1}$ Department of Mathematics, Harvard University, Cambridge, MA
}

\begin{abstract}
We consider a new kind of straight and shifted plane partitions/Young tableaux — ones whose diagrams are no longer of partition shape, but rather Young diagrams with boxes erased from their upper right ends. We find formulas for the number of standard tableaux in certain cases, namely a shifted staircase without the box in its upper right corner, i.e. truncated by a box, a rectangle truncated by a staircase and a rectangle truncated by a square minus a box. The proofs involve finding the generating function of the corresponding plane partitions using interpretations and formulas for sums of restricted Schur functions and their specializations. The number of standard tableaux is then found as a certain limit of this function.
\end{abstract}

Résumé. Nous considérons un nouveau type de partitions planes, ou de tableaux de Young, droits ou décalés, obtenus en privant leurs diagrammes de certaines cellules en haut à droite, et dans certains cas nous trouvons des formules d'énumération pour les tableaux standard. Les preuves impliquent le calcul de la fonction génératrice pour les partitions planes correspondantes, en utilisant des interprétations et des formules pour les sommes de fonctions de Schur restreintes et leurs spécialisations. Le nombre de tableaux standard est alors obtenu comme une certaine limite de cette fonction.

Keywords: plane partitions, tableaux, truncated shapes, hook formulas, Schur functions

\section{Introduction}

In this paper we find product formulas for special cases of a new type of tableaux and plane partitions, ones whose diagrams are not straight or shifted Young diagrams of integer partitions. The diagrams in question are obtained by removing boxes from the north-east corners of a straight or shifted Young diagram and we say that the shape has been truncated by the shape of the boxes removed. We discover formulas for the number of tableaux of specific truncated shapes: shifted staircase truncated by one box in Theorem 1 . rectangle truncated by a staircase shape in Theorem 2 and rectangle truncated by a square minus a box in Theorem 3, these shapes are illustrated as
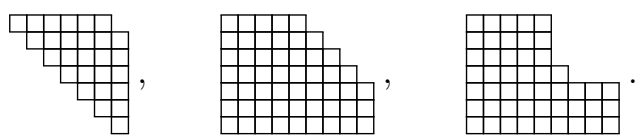

The proofs rely on several steps of interpretations, their details can be found in [Pan10]. Plane partitions of truncated shapes are interpreted as (tuples of) SSYTs, which translates the problem into specializations 
of sums of restricted Schur functions. The number of standard tableaux is found as a polytope volume and then a certain limit of these specializations (generating function for the corresponding plane partitions). The computations involve, among others, complex integration and the Robinson-Schensted-Knuth correspondence.

The consideration of these objects started after R. Adin and Y. Roichman asked for a formula for the number of linear extensions of the poset of triangle-free triangulations, which are equivalent to standard tableaux of shifted straircase shape with upper right corner box removed, [AR]. We find and prove the formula in question as Theorem 1

Theorem 1 The number of shifted standard tableaux of shape $\delta_{n} \backslash \delta_{1}$ is equal to

$$
g_{n} \frac{C_{n} C_{n-2}}{2 C_{2 n-3}},
$$

where $g_{n}=\frac{\left(\begin{array}{c}n+1 \\ 2\end{array}\right) !}{\prod_{0 \leq i<j \leq n}(i+j)}$ is the number of shifted staircase tableaux of shape $(n, n-1, \ldots, 1)$ and $C_{m}=\frac{1}{m+1}\left(\begin{array}{c}2 m \\ m\end{array}\right)$ is the $m-$ th Catalan number. Theorem 2 The number of standard tableaux of truncated straight shape $\underbrace{(n, n, \ldots, n)}_{m} \backslash \delta_{k}$ (assume
$n \leq m$ ), is

$$
\left(m n-\left(\begin{array}{c}
k+1 \\
2
\end{array}\right)\right) ! \times \frac{f_{(n-k-1)^{m}}}{(m(n-k-1)) !} \times \frac{g_{(m, m-1, \ldots, m-k)}}{\left((k+1) m-\left(\begin{array}{c}
k+1 \\
2
\end{array}\right)\right) !} \frac{E_{1}(k+1, m, n-k-1)}{E_{1}(k+1, m, 0)},
$$

where $E_{1}(r, p, s)= \begin{cases}\prod_{r<l<2 p-r+2} \frac{1}{(l+2 s)^{r / 2}} \prod_{2 \leq l \leq r} \frac{1}{((l+2 s)(2 p-l+2+2 s))^{L l / 2\rfloor},}, & \text { r even, } \\ \frac{((r-1) / 2+s) !}{(p-(r-1) / 2+s) !} E_{1}(r-1, p, s), & r \text { odd. }\end{cases}$

Theorem 3 The number of standard truncated tableaux of shape $n^{m} \backslash\left(k^{k-1}, k-1\right)$ is

$$
\frac{\left(n m-k^{2}+1\right) ! f_{\left(m^{n-k}\right)}}{(m(n-k)) !} \frac{f_{\left((m-k)^{k}\right)}}{((m-k) k) !} \frac{\left(k n-k^{2}-k+1\right) !(k(m-k)) !}{\left(m k+k n-2 k^{2}-k+2\right) !} .
$$

We will also exhibit connections with boxed plane partitions, as the generating function we use is the same as the volume generating function for boxed plane partitions. Computer evidence suggests that most truncated shapes do not have product-type formulas.

In [AKR] Adin, King and Roichman have independently found a formula for the case of shifted staircase truncated by one box and rectangle truncated by a square minus a box by methods different from the methods developed here.

\section{Definitions}

We will refer the reader to [Sta99] and [Mac95] for the basic facts and definitions regarding Young tableaux and symmetric functions, which we will use in this paper. Recall the hook-length formulas for the number $f_{\lambda}$ of straight standard Young tableaux (SYT) of shape $\lambda$ and $g_{\lambda}$ for shifted tableaux:

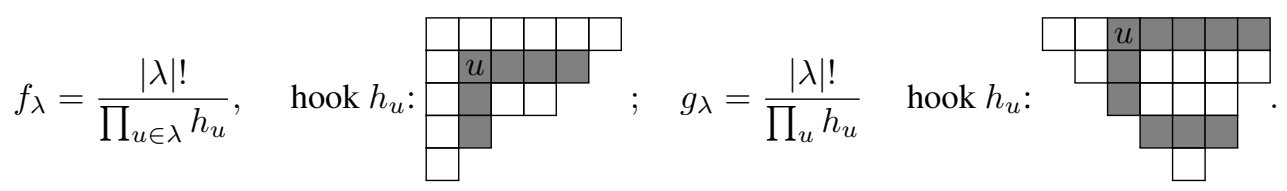


We are now going to define our main objects of study. Let $\lambda=\left(\lambda_{1}, \lambda_{2}, \ldots\right)$ and $\mu=\left(\mu_{1}, \mu_{2}, \ldots\right)$ be integer partitions, such that $\lambda_{i} \geq \mu_{i}$. A straight diagram of truncated shape $\lambda \backslash \mu$ is a left justified array of boxes, such that row $i$ has $\lambda_{i}-\mu_{i}$ boxes. If $\lambda$ has no equal parts we can define a shifted diagram of truncated shape $\lambda \backslash \mu$ as an array of boxes, where row $i$ starts one box to the right of the previous row $i-1$ and has $\lambda_{i}-\mu_{i}$ number of boxes.

We define standard tableaux and plane partitions of truncated shape the usual way except this time they are fillings of truncated diagrams. A standard truncated tableaux of shape $\lambda \backslash \mu$ is a filling of the corresponding truncated diagram with the integers from 1 to $|\lambda|-|\mu|$, such that the entries across rows and down columns are increasing and each number appears exactly once. A plane partition of truncated shape $\lambda \backslash \mu$ is a filling of the corresponding truncated diagram with integers such that they weakly decrease along rows and down columns. For example,

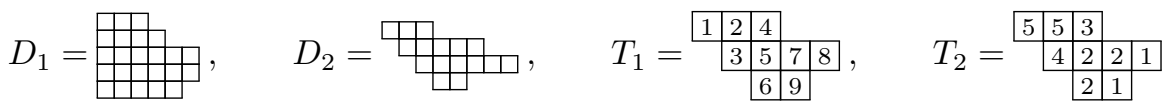

$D_{1}$ is a diagram of straight truncated shape $(6,6,6,6,5) \backslash(3,2) ; D_{2}$ - of shifted truncated shape $(8,7,6,2) \backslash(5,2) ; T_{1}$ and $T_{2}$ are respectively a standard tableaux, and a plane partition (PP) of shifted truncated shape $(5,4,2) \backslash(2)$.

We will denote the staircase partition of size $k$ by $\delta_{k}=(k, k-1, k-2, \ldots, 1)$. Denote by $T[i, j]$ the entry in the box with coordinate $(i, j)$ in the diagram of $T$ with $i$ being the row number and $j-$ the column. Define the generating function for plane partitions of any shape $D$ as

$$
F_{D}(q)=\sum_{T: \operatorname{sh}(T)=D} q^{\sum_{(i, j) \in D} T[i, j]} .
$$

\section{A bijection with skew SSYT}

We will consider a map between truncated plane partitions and skew Semi-Standard Young Tableaux which will enable us to enumerate them using Schur functions.

As a basic setup for this map we first consider truncated shifted plane partitions of staircase shape $\delta_{n} \backslash \delta_{k}$. Let $T$ be such a plane partition. Let $\lambda^{j}=(T[1, j], T[2, j], \ldots, T[n-j, j])$ - the sequence of numbers in the $j$ th diagonal of $T$.

Let $P$ be a reverse skew semi-standard Young tableaux (SSYT) of shape $\lambda^{1} / \lambda^{n-k}$, such that the entries filling the subshape $\lambda^{j} / \lambda^{j+1}$ are equal to $j$, i.e. it corresponds to the sequence $\lambda^{n-k} \subset \lambda^{n-k-1} \subset \cdots \subset$ $\lambda^{1}$. The fact that this is all well defined follows from the inequalities that the $T[i, j]$ 's satisfy by virtue of $T$ being a plane partition, since then $\lambda^{i} / \lambda^{i+1}$ will be horizontal strip.

Define $\phi(T)=P, \phi$ is the bijection in question. Given a reverse skew tableaux $P$ of shape $\lambda \backslash \mu$ and entries smaller than $n$ we can obtain the inverse shifted truncated plane partition $T=\phi^{-1}(P)$ as $T[i, j]=\max (s \mid P[i, s] \geq j)$; if no such entry of $P$ exists let $s=0$.

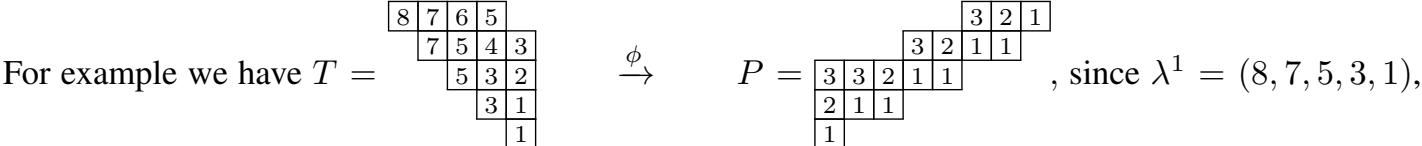

$$
\begin{aligned}
& \lambda^{2}=(7,5,3,1), \lambda^{3}=(6,4,2) \text { and } \lambda^{4}=(5,3) \text {. Notice also that } \\
& \sum T[i, j]=\sum P[i, j]+\left|\lambda^{n-k}\right|(n-k) .
\end{aligned}
$$


The map $\phi$ can be extended to any truncated shape, then the image will be tuples of SSYTs with certain restrictions. For the purposes of this paper we will extend it to truncated plane partitions of shape $\left(n^{m}\right) \backslash \delta_{k}$ as follows. Let $T$ be a plane partition of shape $n^{m} \backslash \delta_{k}$ and assume that $n \leq m$ (otherwise we can reflect about the main diagonal). Let $\lambda=(T[1,1], T[2,2], \ldots, T[n, n]), \mu=(T[1, n-k], T[2, n-$ $k+1], \ldots, T[k+1, n])$ and let $T_{1}$ be the portion of $T$ above and including the main diagonal, hence of shifted truncated shape $\delta_{n} \backslash \delta_{k}$, and $T_{2}$ the transpose of the lower portion including the main diagonal, a shifted PP of shape $(m, m-1, \ldots, m-n+1)$.

Extend $\phi$ to $T$ as $\phi(T)=\left(\phi\left(T_{1}\right), \phi\left(T_{2}\right)\right)$. Here $\phi\left(T_{2}\right)$ is a SSYT of at most $n$ rows (shape $\lambda$ ) and filled with $[1, \ldots, m]$ the same way as in the truncated case. As an example with $n=5, m=6, k=2$ we have

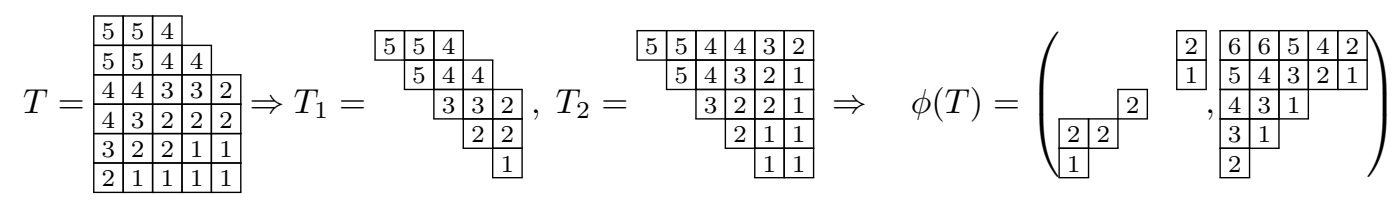

Proposition 1 The map $\phi$ is a bijection between shifted truncated plane partitions $T$ of shape $\delta_{n} \backslash \delta_{k}$ filled with nonnegative integers and (reverse) skew semi-standard Young tableaux with entries in $[1, \ldots, n-$ $k-1]$ of shape $\lambda / \mu$ with $l(\lambda) \leq n$ and $l(\mu) \leq k+1$. Moreover, $\sum_{i, j} T[i, j]=\sum_{i, j} P[i, j]+|\mu|(n-k)$. Similarly $\phi$ is also a bijection between truncated plane partitions $T$ of shape $n^{m} \backslash \delta_{k}$ and pairs of SSYTs $(P, Q)$, such that $\operatorname{sh}(P)=\lambda / \mu, \operatorname{sh}(Q)=\lambda$ with $l(\lambda) \leq n, l(\mu) \leq k+1$ and $P$ is filled with $[1, \ldots, n-$ $k-1], Q$ with $[1, \ldots, m]$. Moreover, $\sum T[i, j]=\sum P[i, j]+\sum Q[i, j]-|\lambda|+|\mu|(n-k)$.

\section{Schur function identities}

We will now consider the relevant symmetric function interpretation arising from the map $\phi$. Substitute the entries $1, \ldots$ in the skew SSYTs in the image with respective variables $x_{1}, \ldots$ and $z_{1}, \ldots$. The idea is to evaluate the resulting expressions at certain finite specializations for $x$ and $z$ (e.g. $x=\left(q, q^{2}, \ldots, q^{n-k-1}\right)$ and $\left.z=\left(1, q, q^{2}, \ldots, q^{m-1}\right)\right)$ to obtain generating functions for the sum of entries in the truncated plane partitions which will later allow us to derive enumerative results.

For the case of shifted truncated shape $\delta_{n} \backslash \delta_{k}$ we have the corresponding sum

$$
S_{n, k}(x ; t)=\sum_{\lambda, \mu \mid l(\lambda) \leq n, l(\mu) \leq k+1} s_{\lambda / \mu}\left(x_{1}, \ldots, x_{n-k-1}\right) t^{|\mu|},
$$

and for the straight truncated shape $n^{m} \backslash \delta_{k}$

$$
D_{n, m, k}(x ; z ; t)=\sum_{\lambda, \mu \mid l(\lambda) \leq n, l(\mu) \leq k+1} s_{\lambda}(z) s_{\lambda / \mu}(x) t^{|\mu|} .
$$

We need to find formulas when $x_{i}=0$ for $i>n-k-1$ and $z_{i}=0$ for $i>m$. Keeping the restriction $l(\mu) \leq k+1$ we have that $s_{\lambda / \mu}(x)=0$ if $l(\lambda)>n$ and this allows us to drop the length restriction on $\lambda$ in both sums.

From now on the different sums will be treated separately. Consider another set of variables $y=$ $\left(y_{1}, \ldots, y_{k+1}\right)$ which together with $\left(x_{1}, \ldots, x_{n-k-1}\right)$ form a set of $n$ variables. Using the determinantal 
formula for the Schur functions, namely that

$$
s_{\nu}\left(u_{1}, \ldots, u_{p}\right)=\frac{a_{\nu+\delta_{p}}(u)}{a_{\delta_{p}}(u)}=\frac{\operatorname{det}\left[u_{i}^{\nu_{j}+p-j}\right]_{i, j=1}^{p}}{\operatorname{det}\left[u_{i}^{p-j}\right]_{i, j=1}^{p}},
$$

in Cauchy's identity for the sum of Schur functions we obtain

$$
\begin{aligned}
\sum_{\lambda, \mu} & s_{\lambda / \mu}\left(x_{1}, \ldots, x_{n-k-1}\right) a_{\mu+\delta_{k+1}}\left(y_{1}, \ldots, y_{k+1}\right) t^{|\mu|} \\
\quad & \prod \frac{1}{1-x_{i}} \prod_{i<j \leq n-k-1} \frac{1}{1-x_{i} x_{j}} \prod_{i<j \leq k+1} \frac{y_{i}-y_{j}}{1-t^{2} y_{i} y_{j}} \prod_{i, j} \frac{1}{1-x_{i} t y_{j}} \prod \frac{1}{1-t y_{i}}
\end{aligned}
$$

We extract the sum $S_{n, k}(x ; t)=\sum_{\lambda, \mu, l(\mu) \leq k+1} s_{\lambda / \mu}(x) t^{|\mu|}$ as the coefficient of $y^{0}$ in the following sum

$$
\left[y^{0}\right]\left(\sum_{\lambda, \mu} s_{\lambda / \mu}(x) a_{\mu+\delta_{k+1}}\left(y_{1}, \ldots, y_{k+1}\right) t^{|\mu|} A\left(y^{-1}\right)\right)=(k+1) ! S_{n, k}(x ; t),
$$

where $A(u)=\sum_{\nu} s_{\nu}(u) a_{\delta_{p}}(u)=\prod \frac{1}{1-u_{i}} \prod_{i<j} \frac{\left(u_{i}-u_{j}\right)}{1-u_{i} u_{j}}$. Since for any doubly infinite series $f(y)$, we have $\left[y^{0}\right] f(y)=\frac{1}{2 \pi i} \int_{C} f(y) y^{-1} d y$, we obtain the following.

Proposition 2 We have that

$$
\begin{aligned}
& S_{n, k}(x ; t)=\frac{(-1)^{\left(\begin{array}{c}
k+1 \\
2
\end{array}\right)}}{(k+1) !} \prod \frac{1}{1-x_{i}} \prod_{i<j \leq n-k-1} \frac{1}{1-x_{i} x_{j}} \\
& \frac{1}{(2 \pi i)^{k+1}} \int_{T} \prod_{i<j \leq k+1} \frac{\left(y_{i}-y_{j}\right)^{2}}{1-t^{2} y_{i} y_{j}} \prod_{i, j} \frac{1}{1-x_{i} t y_{j}} \prod \frac{1}{1-t y_{i}} \prod \frac{1}{y_{i}-1} \prod_{i<j} \frac{1}{y_{i} y_{j}-1} d y_{1} \cdots d y_{k+1},
\end{aligned}
$$

where $T=C_{1} \times C_{2} \times \cdots C_{p}$ and $C_{i}=\left\{z \in \mathbb{C}|| z \mid=1+\epsilon_{i}\right\}$ for $\epsilon_{i}<\left|t^{-1}\right|-1$.

In the case of straight shapes we use Cauchy's identity for the sum of products of Schur functions to obtain

$$
\sum_{\lambda} s_{\lambda}(z) s_{\lambda / \mu}(x)=\prod \frac{1}{1-z_{i} x_{j}} \sum_{\mu} s_{\mu}(z)
$$

Since the length restriction on $\lambda$ becomes redundant when $x=\left(x_{1}, \ldots, x_{n-k-1}\right)$ and $l(\mu) \leq k+1$ we have that

Proposition $3 D_{n, m, k}(x, z ; t)=\prod_{i, j} \frac{1}{1-x_{i} z_{j}}\left(\sum_{\nu \mid l(\nu) \leq k+1} s_{\nu}(z t)\right)$.

For the purpose of enumeration of SYTs we will use this formula as it is. Even though there are formulas, e.g. of Gessel and King, for the sum of Schur functions of restricted length in the form of determinants or infinite sums, they would not give the enumerative answer any more easily. 


\section{A polytope volume as a limit}

Plane partitions of specific shape (truncated or not) of size $N$ can be viewed as integer points in a cone in $\mathbb{R}^{N}$. Let $D$ be the diagram of a plane partition $T$ with $N=|D|$, the coordinates of $\mathbb{R}^{N}$ are indexed by the boxes present in $T$. Then

$C_{D}=\left\{\left(\cdots, x_{i, j}, \cdots\right) \in \mathbb{R}_{\geq 0}^{N}:[i, j] \in D, x_{i, j} \leq x_{i, j+1}\right.$ if $[i, j+1] \in D, x_{i, j} \leq x_{i+1, j}$ if $\left.[i+1, j] \in D\right\}$

is the corresponding cone. Let $P(C)$ be the section of a cone $C$ in $\mathbb{R}_{\geq 0}^{N}$ with the hyperplane $H=$ $\left\{x \mid \sum_{[i, j] \in D} x_{i, j}=1\right\}$. It is easy to see that

$$
\operatorname{Vol}_{N-1}\left(P_{D}\right)=\frac{\# T: S Y T, \operatorname{sh}(T)=D}{N !} \operatorname{Vol}\left(\Delta_{N}\right)
$$

The following lemma, see [Pan10] for proof, helps determine the volume and thus the number of standard tableaux of shape $D$.

Lemma 1 Let $P$ be a $(d-1)$-dimensional rational polytope in $\mathbb{R}_{\geq 0}^{d}$, such that its points satisfy $a_{1}+\cdots+$ $a_{d}=1$ for $\left(a_{1}, \ldots, a_{d}\right) \in P$, and let

$$
F_{P}(q)=\sum_{n} \sum_{\left(a_{1}, \ldots, a_{d}\right) \in n P \cap \mathbb{Z}^{d}} q^{a_{1}+a_{2}+\cdots+a_{d}} .
$$

We have that the $(d-1)$-dimensional volume of $P$ is

$$
\operatorname{Vol}_{d-1}(P)=\left(\lim _{q \rightarrow 1}(1-q)^{d} F_{P}(q)\right) \operatorname{Vol}\left(\Delta_{d}\right),
$$

where $\Delta_{d}$ is the $(d-1)$-dimensional simplex.

Notice that if $P=P\left(C_{D}\right)$ for some shape $D$, then

$$
F_{P}(q)=\sum_{n} \sum_{a \in n P \cap \mathbb{Z}^{N}} q^{n}=\sum_{a \in C_{D} \cap \mathbb{Z}^{N}} q^{|a|}=\sum_{T: P P, \operatorname{sh}(T)=D} q^{\sum T[i, j]}=F_{D}(q) .
$$

Using (10) and this Lemma we get the key fact to enumerating standard tableaux of truncated shapes using evaluations of symmetric functions.

Proposition 4 The number of standard tableaux of shape $D$ is equal to

$$
N ! \lim _{q \rightarrow 1}(1-q)^{N} F_{D}(q)
$$

\section{Shifted staircase truncated by a box: proof of Theorem 1}

We are now going to use Propositions 2 and 4 to find the number of standard shifted tableaux of truncated shape $\delta_{n} \backslash \delta_{1}$. Numerical results show that a product formula for the general case of truncation by $\delta_{k}$ does not exist.

First we will evaluate the integral in Proposition 2 by iteration of the Residue theorem at the possible poles, first with respect to $y_{1}$, then $y_{2}$. We obtain

$$
S_{n, 1}(x ; t)=\frac{1}{2} \prod \frac{1}{1-x_{i}} \prod_{1 \leq i<j \leq n-k-1} \frac{1}{1-x_{i} x_{j}} \sum_{i \geq 0} \frac{1}{1-t^{2}}\left(1+u_{i}\right)^{2} u_{i}^{n-3} \prod_{j \neq i} \frac{1}{u_{i}-u_{j}} \prod_{j \geq 0} \frac{1}{1-u_{i} u_{j}}
$$


where $u_{i}=t x_{i}$ with $x_{0}=1$. Using the partial fraction version of the determinantal formula (6) for $s_{s-p+1}(u)$ we can simplify the sum above as

$$
\sum_{i=0}^{n-k-1} \frac{u_{i}^{s}}{\prod\left(u_{i}-u_{j}\right)} \prod \frac{1}{1-u_{i} u_{j}}=\sum_{p \geq 0} h_{s-n+k+1+p}(u) h_{p}(u)=c_{s-n+k+1}(u),
$$

where $c_{i}=\sum_{n \geq 0} h_{n} h_{n+i}$. We then have the new formulas

$$
S_{n, 1}(x ; t)=\prod \frac{1}{1-x_{i}} \prod_{1 \leq i<j \leq n-k-1} \frac{1}{1-x_{i} x_{j}} \frac{1}{1-t^{2}}\left(c_{1}(u)+c_{0}(u)\right)
$$

Proof of Theorem 1; We will use Proposition 4 and the formula (12). For the shape $D=\delta_{n} \backslash \delta_{1}$ we have,

$$
F_{D}(q)=\sum_{T \mid \operatorname{sh}(T)=D} q^{\sum T[i, j]}=\sum_{P=\phi(T)} q^{(n-1)|\mu|+\sum P[i, j]}=S_{n, 1}\left(q, q^{2}, \ldots, q^{n-2} ; q^{n-1}\right) .
$$

In order to find $\lim _{q \rightarrow 1}(1-q)\left(\begin{array}{c}n+1 \\ 2\end{array}\right)-1 F_{D}(q)$, using the formula $\sqrt{12}$, we need to find $\lim _{q \rightarrow 1}(1-$ $q)^{2 n-3} c_{s}(u)$ for $u=\left(q^{n-1}, \ldots, q^{2 n-3}\right)$. Let $c_{s}(x ; y)=\sum_{l} h_{l}(x) h_{l+s}(y)$ where $x=\left(x_{1}, \ldots, x_{n}\right)$ and $y=\left(y_{1}, \ldots, y_{m}\right)$. We have that

$$
\begin{aligned}
c_{s}(x ; y) & =\sum_{l} \sum_{i_{1} \leq \cdots \leq i_{l} ; j_{1} \leq \cdots \leq j_{l+s}} x_{i_{1}} \cdots x_{i_{l}} y_{j_{1}} \cdots y_{j_{s+l}} \\
& =\sum_{p} h_{s}\left(y_{1}, \ldots, y_{p}\right) \sum_{P:(1, p) \rightarrow(n, m)}(-1)^{m+n-p-\# P} \sum_{l} h_{l}\left((x y)_{P}\right),
\end{aligned}
$$

where the sum runs over all fully ordered collections of lattice points $P$ in between $(1, p)$ to $(n, m)$ and $(x y)_{P}=\left(x_{i_{1}} y_{j_{1}}, \ldots\right)$ for $\left(i_{1}, j_{1}\right), \ldots \in P$ and the $(-1) \mathrm{s}$ indicate the underlying inclusion-exclusion process. We also have that

$$
\sum_{l} h_{l}\left((x y)_{P}\right)=\frac{1}{\prod_{(i, j) \in P}\left(1-x_{i} y_{j}\right)} .
$$

The degree of $1-q$ dividing the denominators after substituting $\left(x_{i}, y_{j}\right)=\left(u_{i}, u_{j}\right)=\left(q^{n-2+i}, q^{n-2+j}\right)$ for the evaluation of $c_{s}(u)$ is equal to the number of points in $P$. \#P is maximal when the lattice path is from $(1,1)$ to $(n-1, n-1)$ and is saturated, so $\max (\# P)=2(n-1)-1=2 n-3$. The other summands will contribute 0 when multiplied by the larger power of $(1-q)$ and the limit is taken. For each maximal path we have $\{i+j \mid(i, j) \in P\}=\{2, \ldots, 2 n-2\}$ and the number of these paths is $\left(\begin{array}{c}2 n-4 \\ n-2\end{array}\right)$, so

$$
(1-q)^{2 n-3} c_{s}\left(q^{n-1}, \ldots, q^{2 n-3}\right)=\left(\begin{array}{c}
2 n-4 \\
n-2
\end{array}\right) \prod_{i=2}^{2 n-2} \frac{1-q}{1-q^{2(n-2)+i}}+(1-q) \ldots
$$

where the remaining terms are divisible by $1-q$, hence contribute 0 when the limit is taken. 
Now we can proceed to compute $\lim _{q \rightarrow 1}(1-q)^{N} S_{n, 1}\left(q^{1}, q^{2}, \ldots, ; q^{n-1}\right)$. Putting all these together we have that

$$
\begin{aligned}
& \lim _{q \rightarrow 1}(1-q)^{\left(\begin{array}{c}
n+1 \\
2
\end{array}\right)-1} S_{n, 1}\left(q^{1}, \ldots, q^{n-2} ; q^{n-1}\right) \\
& =\prod_{0 \leq i<j \leq n-2} \frac{1}{i+j} \frac{1}{2(n-1)} 2\left(\begin{array}{c}
2 n-4 \\
n-2
\end{array}\right) \prod_{i=2}^{2 n-2} \frac{1}{2 n-4+i}=\frac{g_{\delta_{n-2}}}{\left(\begin{array}{c}
n-1 \\
2
\end{array}\right) !} \frac{1}{(n-1)}\left(\begin{array}{c}
2 n-4 \\
n-2
\end{array}\right) \frac{(2 n-3) !}{(4 n-6) !},
\end{aligned}
$$

where $g_{n-2}=\frac{\left(\begin{array}{c}n-1 \\ 2\end{array}\right) !}{\prod_{0 \leq i<j \leq n-2}(i+j)}$ is the number of shifted staircase tableaux of shape $(n-2, \ldots, 1)$. After algebraic manipulations we arrive at the desired formula.

\section{Rectangle truncated by staircase: proof of Theorem 2}

We will compute the number of standard tableaux of straight truncated shape $D=n^{m} \backslash \delta_{k}$. By Propositions 1 and the definition of $D_{n, m, k}(x ; z ; t)$ in equation (5) we have that

$$
F_{n^{m} \backslash \delta_{k}}(q)=D_{n, m, k}\left(q, q^{2}, \ldots, q^{n-k-1} ; 1, q, \ldots, q^{m-1} ; q^{n-k}\right) .
$$

Using the simplified almost-product type formula for $D_{n, m, k}$ from Proposition 3 , the number of standard tableaux of shape $n^{m} \backslash \delta_{k}$ by Proposition 4 is

$$
\begin{aligned}
& \lim _{q \rightarrow 1}(1-q)^{n m-\left(\begin{array}{c}
k+1 \\
2
\end{array}\right)} F_{D}(q) \\
& =\lim _{q \rightarrow 1}\left(\prod_{i=1, j=0}^{n-k-1, m-1} \frac{1-q}{1-q^{i+j}}(1-q)^{m(k+1)-\left(\begin{array}{c}
k+1 \\
2
\end{array}\right)}\left(\sum_{\nu \mid l(\nu) \leq k+1} s_{\nu}\left(q^{n-k}, q^{n-k+1}, \ldots, q^{m-1+n-k}\right)\right)\right) .
\end{aligned}
$$

We are thus going to compute the last factor.

Lemma 2 Let $p \geq r$ and $N=r p-\left(\begin{array}{l}r \\ 2\end{array}\right)$. Then for any $s$ we have

$$
\lim _{q \rightarrow 1}(1-q)^{N} \sum_{\lambda \mid l(\lambda) \leq r} s_{\lambda}\left(q^{1+s}, \ldots, q^{p+s}\right)=\frac{g_{(p, p-1, \ldots, p-r+1)}}{N !} \frac{E_{1}(r, p, s)}{E_{1}(r, p, 0)},
$$

where

$$
E_{1}(r, p, s)=\prod_{r<l<2 p-r+2} \frac{1}{(l+2 s)^{r / 2}} \prod_{2 \leq l \leq r} \frac{1}{((l+2 s)(2 p-l+2+2 s))^{\lfloor l / 2\rfloor}}
$$

for $r$ even and $E_{1}(r, p, s)=\frac{((r-1) / 2+s) !}{(p-(r-1) / 2+s) !} E_{1}(r-1, p, s)$ when $r$ is odd and $g_{\lambda}$ is the number of shifted SYTs of shape $\lambda$.

Proof: Consider the Robinson-Schensted-Knuth (RSK) correspondence between SSYTs with no more than $r$ rows filled with $x_{1}, \ldots, x_{p}$ and symmetric $p \times p$ integer matrices $A$. The limit on the number of rows translates through Schensted's theorem to the fact that there are no $m+1$ nonzero entries in $A$ with coordinates $\left(i_{1}, j_{1}\right), \ldots,\left(i_{r+1}, j_{r+1}\right)$, s.t. $i_{1}<\cdots<i_{r+1}$ and $j_{1}>\cdots>j_{r+1}$ (i.e. a decreasing 
subsequence of length $r+1$ in the generalized permutation corresponding to $A$ ). Let $\mathcal{A}$ be the set of such matrices. Let $\mathcal{A}, \subset \mathcal{A}$ be the set of $0-1$ matrices satisfying this condition, we will refer to them as allowed configurations. Notice that $A \in \mathcal{A}$ if and only if $B \in \mathcal{B}$, where $B[i, j]=\left\{\begin{array}{l}1, \text { if } A[i, j] \neq 0 \\ 0, \text { if } A[i, j]=0\end{array}\right.$. We thus have that

$$
\begin{aligned}
\sum_{\lambda \mid l(\lambda) \leq r} s_{\lambda}\left(x_{1}, \ldots, x_{p}\right) & =\sum_{A \in \mathcal{A}} \prod_{i} x_{i}^{A[i, i]} \prod_{i>j}\left(x_{i} x_{j}\right)^{A[i, j]} \\
& =\sum_{B \in \mathcal{B}} \prod_{i: B[i, i]=1}\left(\sum_{a_{i, i}=1}^{\infty} x_{i}^{a_{i, i}}\right) \prod_{i>j: B[i, j]=1}\left(\sum_{a_{i, j}=1}^{\infty}\left(x_{i} x_{j}\right)^{a_{i, j}}\right) \\
& =\sum_{B \in \mathcal{B}} \prod_{i: B[i, i]=1} \frac{x_{i}}{1-x_{i}} \prod_{i>j: B[i, j]=1} \frac{x_{i} x_{j}}{1-x_{i} x_{j}} .
\end{aligned}
$$

Notice that $B$ cannot have more than $N$ nonzero entries on or above the main diagonal. No diagonal $i+j=l$ (i.e. the antidiagonals) can have more than $r$ nonzero entries on it because of the longest decreasing subsequence condition. Also if $l \leq r$ or $l>2 p-r+1$, the total number of points on such diagonal are $l-1$ and $2 p-l+1$ respectively. Since $B$ is also symmetric the antidiagonals $i+j=l$ will have $r-1$ entries if $l \equiv r-1(\bmod 2)$ and $r$ is odd. Counting the nonzero entries on each antidiagonal on or above the main diagonal gives always exactly $N$ in each case for the parity of $r$ and $p$.

If $B$ has less than $N$ nonzero entries, then

$$
\begin{gathered}
\lim _{q \rightarrow 1}(1-q)^{N} \prod_{i: B[i, i]=1} \frac{q^{i+1}}{1-q^{i+s}} \prod_{i>j: B[i, j]=1} \frac{q^{i+j+2 s}}{1-q^{i+j+2 s}}= \\
\lim _{q \rightarrow 1}(1-q)^{N-|B|>0} \prod_{i: B[i, i]=1} \frac{q^{i+1}(1-q)}{1-q^{i+s}} \prod_{i>j: B[i, j]=1} \frac{q^{i+j+2 s}(1-q)}{1-q^{i+j+2 s}}=0,
\end{gathered}
$$

so such $B$ s won't contribute to the final answer.

Consider now only $B$ s with maximal possible number of nonzero entries (i.e. $N$ ), which forces them to have exactly $r$ (or $r-1$ ) nonzero entries on every diagonal $i+j=l$ for $r<l \leq 2 p-r+1$ and all entries in $i+j \leq r$ and $i+j>2 p-r+1$.

If $r$ is even, then there are no entries on the main diagonal when $r<l<2 p-r+2$ and so there are $r / 2$ terms on each diagonal $i+j=l$. Thus every such $B$ contributes the same factor when evaluated at $x=\left(q^{1+s}, \ldots\right)$ :

$$
E_{q}(r, p, s):=\prod_{r<l<2 p-r+2} \frac{q^{(l+2 s) r / 2}}{\left(1-q^{l+2 s}\right)^{r / 2}} \prod_{2 \leq l \leq r} \frac{q^{(l+4 s+2 p-l+2)\lfloor l / 2\rfloor}}{\left(\left(1-q^{l+2 s}\right)\left(1-q^{2 p-l+2+2 s}\right)\right)^{\lfloor l / 2\rfloor}} .
$$

If $r$ is odd, then the entries on the main diagonal will all be present with the rest being as in the even case with $r-1$, so the contribution is

$$
E_{q}(r, p, s):=\prod_{\frac{r+1}{2} \leq i \leq p-\frac{r+1}{2}+1} \frac{q^{i+s}}{1-q^{i+s}} E_{q}(r-1, p, s) .
$$


Let now $M$ be the number of such maximal $B \mathrm{~s}$ in $\mathcal{A}_{0}$. The final answer after taking the limit is $M E_{1}(r, p, s)$, where we have that $E_{1}(r, p, s)=\lim _{q \rightarrow 1}(1-q)^{N} E_{q}(r, p, s)$ as defined in the statement of the lemma.

In order to find $M$ observe that the case of $s=0$ gives

$$
\lim _{q \rightarrow 1}(1-q)^{N} \sum_{\lambda \mid l(\lambda) \leq r} s_{\lambda}\left(q^{1}, \ldots, q^{p}\right)=M E_{1}(r, p, 0),
$$

on one hand. On the other hand via the bijection $\phi$ we have that

$$
\sum_{\lambda \mid l(\lambda) \leq m} s_{\lambda}\left(q^{1}, \ldots, q^{n}\right)=\sum_{T} q^{\sum T[i, j]}
$$

where the sum on the right goes over all shifted plane partitions $T$ of shape $(p, p-1, \ldots, p-r+1)$. Multiplying by $(1-q)^{N}$ and taking the limit on the right hand side gives us, by the inverse of Proposition 4 . $\frac{1}{N !}$ times the number of standard shifted tableaux of that shape. This number is well known and is

$g_{(p, p-1, \ldots, p-r+1)}=\frac{N !}{\prod_{u} h_{u}}$, where the product runs over the hooklengths of all boxes on or above the main diagonal of $\left(p^{r}, r^{p-r}\right)$. Putting all this together gives

$$
M E_{1}(r, p, 0)=\frac{g_{(p, p-1, \ldots, p-r+1)}}{N !} .
$$

Solving for $M$ we obtain the final answer:

$$
\frac{g_{(p, p-1, \ldots, p-r+1)}}{N !} \frac{E_{1}(r, p, s)}{E_{1}(r, p, 0)} .
$$

Proof of Theorem 2; Take the limit in equation (13), by using Lemma 2 with $r=k+1, s=n-k-1$ and $p=m-2$.

\section{Truncation by almost squares: proof of Theorem 3}

We will apply our methods to count standard tableaux, whose shape is a rectangle truncated by a square without a corner. Specifically, let $D=n^{m} \backslash\left(k^{k-1}, k-1\right)$ be such a truncated rectangle. As in the previous case we can assume that $n<m$. Let $2 k \leq n+1$. For any plane partition $T$ of shape $D$, let $p=T[k, n-k+1]$ be the value of the entry in that missing square corner. Because of the row and column inequalities, $T$ is in bijection with $T^{\prime}$ of straight truncated shape $n^{m} \backslash \delta_{k-1}$, where the $T^{\prime}[i, j]=T[i, j]$ for $(i, j) \in D$ and $T^{\prime}[i, j]=T[k, n-k+1]=p$ for the values in the extra boxes. For example,

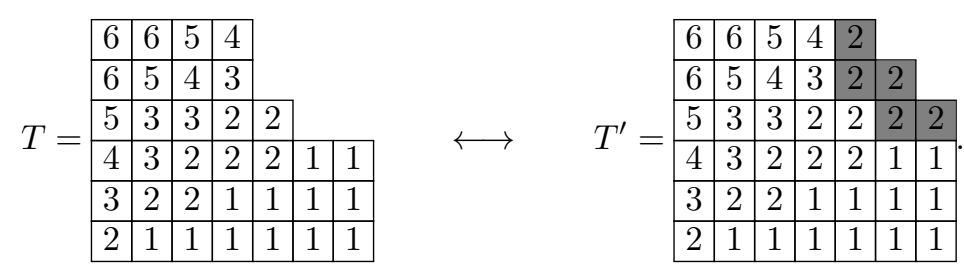


Then the generating function $F_{n^{m} \backslash\left(k^{k-1}, k-1\right)}(q)$ is obtained, using equation 9 , from

$$
H_{n, m, k}(z ; x ; t)=\sum_{p} \sum_{\lambda} s_{\lambda}(z) s_{\lambda /\left(p^{k}\right)}(x) t^{p}=\prod \frac{1}{1-z_{i} x_{j}} \sum_{p} s_{\left(p^{k}\right)}(z) t^{p},
$$

by substituting $x=\left(q, q^{2}, \ldots, q^{n-k}\right), z=\left(1, q, \ldots, q^{m-1}\right)$ as in the case of the rectangle truncated by a staircase $n^{m} \backslash \delta_{k-1}$. For the value of $t$ in this case, since $\sum T[i, j]=\sum T^{\prime}[i, j]-p\left(\left(\begin{array}{c}k+1 \\ 2\end{array}\right)-1\right)$ to account

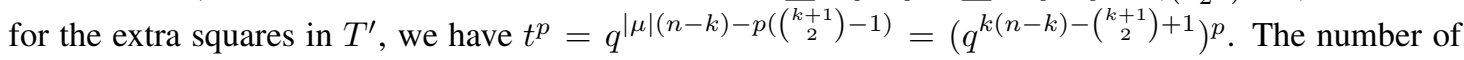
standard truncated tableaux of this shape will then be given by

$$
\lim _{q \rightarrow 1}(1-q)^{n m-k^{2}+1} H_{n, m, k}\left(1, q, \ldots, q^{m-1} ; q, q^{2}, \ldots, q^{n-k} ; q^{k(n-k)-\left(\begin{array}{c}
k+1 \\
2
\end{array}\right)+1}\right) .
$$

We will now evaluate the sum of $s_{\left(p^{k}\right)}$ over $p$ and the relevant limit.

Lemma 3 Let $f_{q}(v)=v^{\left(\begin{array}{c}k \\ 2\end{array}\right)} \prod_{i \leq k<j}\left(v q^{m-i}-q^{m-j}\right)=\sum a_{i}(q) v^{i}$, then

$$
\sum_{p} s_{\left(p^{k}\right)}\left(1, \ldots, q^{m-1}\right) t^{p}=\prod_{m-k \leq i<m ; 0 \leq j<m-k} \frac{1}{\left(q^{i}-q^{j}\right)} \sum_{i} a_{i}(q) \frac{1}{1-q^{i} t} .
$$

If $t=q^{s}$, then

$$
\lim _{q \rightarrow 1}(1-q)^{m k-k^{2}+1} \sum_{p} s_{\left(p^{k}\right)}\left(1, \ldots, q^{m-1}\right)\left(q^{s}\right)^{p}=\prod_{i=m-k ; j=0}^{m-1 ; m-k-1} \frac{1}{(j-i)} \frac{\left(\left(\begin{array}{l}
k \\
2
\end{array}\right)+s\right) !(k(m-k)) !}{\left(m k-\left(\begin{array}{c}
k+1 \\
2
\end{array}\right)+s+1\right) !}
$$

Proof outline: We apply the determinantal formula for the Schur functions 6 to $s_{(p)}\left(1, \ldots, q^{m-1}\right)$, which in this case reduces to a $m \times m$ Vandermonde determinant. and hence a polynomial in $v=q^{p}$. Summing over $p$ gives the first part of the lemma. Taking the limit of the resulting expression reduces the computation to certain integral of $f(v)$.

Proof of Theorem 3: For the number of standard tableaux of shape $D$ take $\lim _{q \rightarrow 1}(1-q)^{n m-k^{2}+1} F_{D}(q)$, using the formula for $F_{D}(q)$ from 16, formula [15), and applying the second part of the Lemma with $s=k(n-k)-\left(\begin{array}{c}k+1 \\ 2\end{array}\right)+1$. We can simplify the products by viewing them as products of hook-lengths and invoking the hook-length formula for the respective shape.

\section{Boxed plane partitions}

The boxed plane partition corresponding to a plane partition $T$ of shape $D$ is a $3 \mathrm{D}$ diagram, where the base in the $x y$ plane is $D$ and on top of each square $[i, j] \in D$ there are $T[i, j]$ unit cubes. Its volume is the number of cubes, i.e. $\sum T[i, j]$, so our generating function $F_{D}(q)$ is the same as the volume generating function for boxed plane partitions of shape $D$. If $D=n^{n}$ and $T[1,1]=n$, as $n \rightarrow \infty$, such boxed plane partitions approximate stepped surfaces, as the picture on the right shows. 

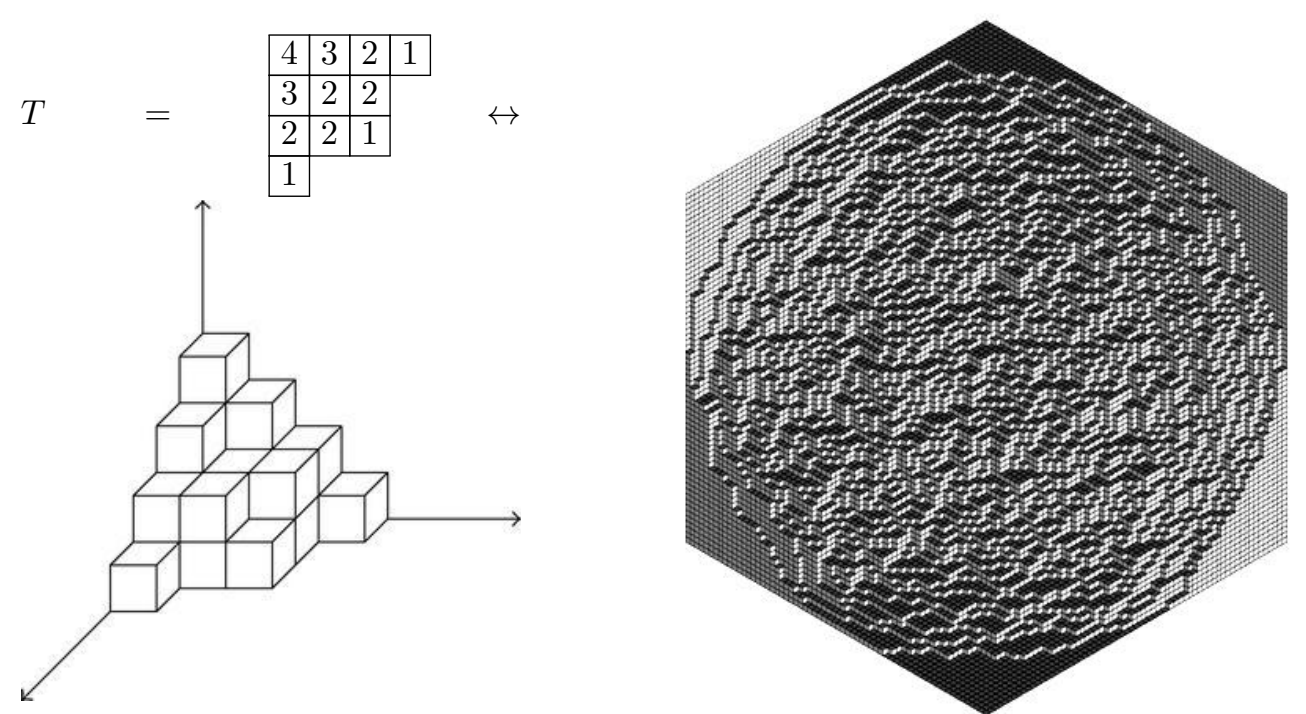

Using the methods developed so far, in particular equation (9), we can easily derive volume generating functions for boxed plane partitions with certain restrictions.

Proposition 5 The average value under the volume statistic of boxed plane partitions of base $\left(n^{n}\right)$ and whose corner is at a fixed value $m$, i.e. $T[1, n]=m$, is given by

$$
\frac{\sum_{T: \operatorname{sh}(T)=n^{n} ; T_{1 n}=m} q^{\sum T_{i j}}}{\sum_{T: \operatorname{sh}(T)=n^{n}} q^{\sum T_{i j}}}=\prod_{i=1}^{n}\left(1-q^{n-1+i}\right)\left[\begin{array}{c}
n+m-1 \\
m
\end{array}\right]_{q} .
$$

More generally, the average value under the volume statistic of boxed partitions on $\left(n^{n}\right)$, whose $n-k$ 'th diagonal is fixed at a certain value $\mu$, i.e. $(T[1, n-k+1], T[2, n-k+2], \ldots)=\mu$, is

$$
\begin{aligned}
& \frac{\sum_{T: \operatorname{sh}(T)=n^{n} ;(T[1, n-k+1], \ldots, T[k, n])=\mu} q^{\sum T[i, j]}}{\sum_{\operatorname{sh}(T)=n^{n}} q^{\sum T[i, j]}}=\prod_{0<i<n+1, n-k<j<n+1} \frac{1}{1-q^{i+j-1}} \times \\
& \times q^{(n-k+1)|\mu|} \frac{\prod_{0<i<j<n+1}\left(q^{\mu_{i}+n-i}-q^{\mu_{j}+n-j}\right)}{\prod_{0<i<j<n+1}\left(q^{j}-q^{i}\right)} \frac{\prod_{0<i<j<k+1}\left(q^{\mu_{i}+k-i}-q^{\mu_{j}+k-j}\right)}{\prod_{0 \leq i<j \leq k}\left(q^{j}-q^{i}\right)} .
\end{aligned}
$$

\section{References}

[AR] R. Adin and Y Roichman, Triangle-Free Triangulations, Hyperplane Arrangements and Shifted Tableaux.arXiv:1009.2628v1

[AKR] R. Adin, R. King, and Y. Roichman, Enumeration of standard Young tableaux of certain truncated shapes.arXiv:1011.0366v1

[Mac95] I. G. Macdonald, Symmetric functions and Hall polynomials, 2nd ed., Oxford Mathematical Monographs, The Clarendon Press Oxford University Press, New York, 1995.

[Pan10] Greta Panova, Tableaux and plane partitions of truncated shapes (2010).arXiv:1011.0795

[Sta99] Richard P. Stanley, Enumerative combinatorics. Vol. 2, Cambridge Studies in Advanced Mathematics, vol. 62, Cambridge University Press, Cambridge, 1999. 